\title{
Inspiration from intersecting D-branes: general supersymmetry breaking soft terms in no-scale $\mathcal{F}$-SU(5)
}

\author{
Ron De Benedetti ${ }^{1}$, Chuang $\mathbf{L i}^{2,3}$, Tianjun $\mathbf{L i}^{2,3}$, Adam Lux ${ }^{4}$, James A. Maxin ${ }^{1, a}$, Dimitri V. Nanopoulos ${ }^{5,6,7}$ \\ ${ }^{1}$ Department of Chemistry and Physics, Louisiana State University, Shreveport, LA 71115, USA \\ ${ }^{2}$ CAS Key Laboratory of Theoretical Physics, Institute of Theoretical Physics, Chinese Academy of Sciences, Beijing 100190, China \\ ${ }^{3}$ School of Physical Sciences, University of Chinese Academy of Sciences, No. 19A Yuquan Road, Beijing 100049, China \\ ${ }^{4}$ Department of Physics and Engineering Physics, The University of Tulsa, Tulsa, OK 74104, USA \\ ${ }^{5}$ George P. and Cynthia W. Mitchell Institute for Fundamental Physics and Astronomy, Texas A\&M University, College Station, TX 77843, USA \\ ${ }^{6}$ Astroparticle Physics Group, Houston Advanced Research Center (HARC), Mitchell Campus, Woodlands, TX 77381, USA \\ ${ }^{7}$ Division of Natural Sciences, Academy of Athens, 28 Panepistimiou Avenue, 10679 Athens, Greece
}

Received: 28 September 2018 / Accepted: 10 November 2018 / Published online: 21 November 2018

(c) The Author(s) 2018

\begin{abstract}
Motivated by D-brane model building, we evaluate the $\mathcal{F}-S U(5)$ model with additional vector-like particle multiplets, referred to as flippons, within the framework of No-Scale Supergravity with non-vanishing general supersymmetry breaking soft terms at the string scale. The viable phenomenology is uncovered by applying all current experimental constraints, including but not limited to the correct light Higgs boson mass, WMAP and Planck relic density measurements, and several LHC constraints on supersymmetric particle spectra. Four interesting regions of the parameter space arise, as well as mixed scenarios, given by: (1) light stop coannihilation; (2) pure Higgsino dark matter; (3) Higgs funnel; and (4) light stau coannihilation. All regions can generate the observed value of the relic density commensurate with a $125 \mathrm{GeV}$ light Higgs boson mass, with the exception of the relatively small relic density value for the pure Higgsino lightest supersymmetric particle. This work is concluded by gauging the model against present LHC search constraints and derivation of the final states observable at the LHC for each of these scenarios.
\end{abstract}

\section{Introduction}

Successful confirmation of the Standard Model (SM) was celebrated when the lightest CP-even Higgs boson with mass $m_{h}=125.09 \pm 0.24 \mathrm{GeV}$ was discovered at the LHC in $2012[1,2]$. Despite the memorable occasion, severe anomalies persisted in the SM, for instance, the gauge hierarchy problem, conspicuous absence of gauge coupling unification, and lack of a plausible dark matter candidate, just to highlight

\footnotetext{
a e-mail: james.maxin@1sus.edu
}

a few. Beyond the SM (BSM) theories, and supersymmetry in particular, can rescue high-energy physics from these SM deficiencies. Supersymmetry (SUSY) can solve the gauge hierarchy problem and produce gauge coupling unification. In supersymmetric SMs (SSMs), the large top quark Yukawa coupling can radiatively break electroweak (EW) gauge symmetry. The lightest supersymmetric particle (LSP) neutralino $\left(\widetilde{\chi}_{1}^{0}\right)$ can serve as a viable dark matter candidate in SSMs with $R$-parity. Of particular significance, gauge coupling unification strongly suggests Grand Unified Theories (GUTs), and SUSY GUTs can be elegantly constructed from superstring theory. Supersymmetry thus provides rather auspicious new physics beyond the SM, beautifully merging low energy phenomenology with high-energy fundamental physics. Despite the successful intervention SUSY can inject into high-energy physics, it remains considerably challenging to obtain a light Higgs boson mass around $125 \mathrm{GeV}$ in the Minimal SSM (MSSM) without embracing either multi-TeV top squarks with small mixing or $\mathrm{TeV}$-scale top squarks with large mixing [3]. Compounding the effort are the strong constraints on the SSM viable parameter space established by the LHC SUSY searches. Principally among those LHC constraints are the masses for the gluino $(\tilde{g})$ and light stop $\left(\tilde{t}_{1}\right)$, where the nominal exclusion curves imply the masses are heavier than about $1.9 \mathrm{TeV}$ and $900 \mathrm{GeV}$, respectively [4], indicating that an electroweak fine-tuning problem may potentially lurk in SUSY.

The aforementioned threats to developing effective BSM constructions notwithstanding, string theory perseveres as one of the most promising theories for quantum gravity. As opposed to the conventional unification at the GUT scale realized in the typical SUSY GUT, we proposed the testable flipped $S U(5) \times U(1)_{X}$ models [5-7] with addi- 
tional TeV-scale vector-like particles [8], which we colorfully dubbed flippons, to obtain gauge coupling unification at the string scale. Subsequently, we further constructed this class of flipped $S U(5)$ models from local F-theory model building $[9,10]$. These flipped $S U(5)$ models with extra vector-like multiplets can be realized in free-fermionic string constructions also [11], hence we denoted them $\mathcal{F}-S U(5)$. Let's enumerate the "Miracles" [12] of the flippons in $\mathcal{F}$-SU (5):

(1) The lightest CP-even Higgs boson mass can be easily lifted to $125 \mathrm{GeV}$ due to one-loop contributions from the Yukawa couplings between the flippons and Higgs fields $[12,13]$.

(2) It is well-known that dimension-five proton decays mediated by colored Higgsinos are highly suppressed due to the missing partner mechanism and $\mathrm{TeV}$-scale $\mu$ term in the flipped $S U(5) \times U(1)_{X}$ models. In $\mathcal{F}-S U(5)$, the $S U(3)_{C} \times S U(2)_{L}$ gauge couplings do in fact unify at the traditional GUT scale while the two unified gauge couplings increase as a result of the vector-like particle contributions $[14,15]$. Therefore, dimension-six proton decays via heavy gauge boson exchanges are within the reach of the future proton decay experiments such as the Hyper-Kamiokande experiment. More concisely, $\mathcal{F}$ $S U(5)$ models differ from the minimal flipped $S U(5) \times$ $U(1)_{X}$ model since the proton lifetime in the minimal model is too lengthy for future proton decay experiments.

(3) The lightest neutralino serves as the LSP and is lighter than the light stau attributable to the longer running of the Renormalization Group Equations (RGEs) in No-Scale supergravity [16], allowing the LSP neutralino to prevail as a dark matter candidate [17-19]. More acutely, No-Scale $\mathcal{F}-S U(5)$ yields the uncommon mass hierarchy $M\left(\tilde{t}_{1}\right)<M(\tilde{g})<M(\tilde{q})$ of a light stop and gluino both substantially lighter than all other squarks $(\tilde{q})[17-$ 19]. The net effect of this rare SUSY spectrum is the production of four top quarks, leading to large multijet events at the LHC [20].

(4) An alliance between No-Scale supergravity and the Giudice-Masiero (GM) mechanism [21] allows the SUSY electroweak fine-tuning problem to be resolved naturally $[22,23]$.

Several prior No-Scale $\mathcal{F}$-SU (5) analyses have intimately examined vanishing SUSY breaking soft terms with the exception of a single unified gaugino parameter $M_{1 / 2}$ (see, for example, Refs. [12,24,25]). To complement those prior sweeping studies, we now consider in this paper non-zero general SUSY breaking soft terms in No-Scale $\mathcal{F}-S U(5)$, partially inspired by D-brane model building [26]. The lowenergy particle spectra will be examined that are consistent with all the current experimental constraints, and interesting diverse regions of the parameter space that can generate the observed dark matter relic density and correct light Higgs boson mass, in addition to several other currently operating experiments, will be identified and analyzed discretely to derive low-energy phenomenology. Finally, benchmarks models will be classified and branching fractions computed to itemize the final states observable at the LHC.

\section{The $\mathcal{F}-S U(5)$ model}

Here we only briefly review the minimal flipped $S U(5)$ model [5-7], where the gauge group $S U(5) \times U(1)_{X}$ can be embedded into the $S O(10)$ model. More comprehensive discussions of the minimal flipped $S U$ (5) model can be found in Refs. [12,20,23,24,27] and references therein. We define the generator $U(1)_{Y^{\prime}}$ in $S U(5)$ as

$T_{\mathrm{U}(1)_{\mathrm{Y}^{\prime}}}=\operatorname{diag}\left(-\frac{1}{3},-\frac{1}{3},-\frac{1}{3}, \frac{1}{2}, \frac{1}{2}\right)$,

and subsequently the hypercharge is given by

$Q_{Y}=\frac{1}{5}\left(Q_{X}-Q_{Y^{\prime}}\right)$.

We have three families of the SM fermions whose quantum numbers under $S U(5) \times U(1)_{X}$ are respectively

$F_{i}=(\mathbf{1 0}, \mathbf{1}), \bar{f}_{i}=(\overline{\mathbf{5}},-\mathbf{3}), \bar{l}_{i}=(\mathbf{1}, \mathbf{5})$,

where $i=1,2,3$. The SM particle assignments in $F_{i}, \bar{f}_{i}$ and $\bar{l}_{i}$ are

$F_{i}=\left(Q_{i}, D_{i}^{c}, N_{i}^{c}\right), \bar{f}_{i}=\left(U_{i}^{c}, L_{i}\right), \bar{l}_{i}=E_{i}^{c}$,

where $Q_{i}, U_{i}^{c}, D_{i}^{c}, L_{i}, E_{i}^{c}$ and $N_{i}^{c}$ are the left-handed quark doublets, right-handed up-type quarks, down-type quarks, left-handed lepton doublets, right-handed charged leptons, and neutrinos, respectively. Generation of the heavy righthanded neutrino masses is accomplished by introduction of three SM singlets $\phi_{i}$.

Breaking the GUT and electroweak gauge symmetries is achieved via introduction of the two pairs of Higgs representations

$$
\begin{aligned}
H & =(\mathbf{1 0}, \mathbf{1}), \bar{H}=(\overline{\mathbf{1 0}},-\mathbf{1}), \\
h & =(\mathbf{5},-\mathbf{2}), \bar{h}=(\overline{\mathbf{5}}, \mathbf{2}) .
\end{aligned}
$$

The states in the $H$ multiplet are labeled by the same symbols as in the $F$ multiplet, and for $\bar{H}$ we merely add "bar" above the fields. Explicitly, the Higgs particles are

$$
\begin{aligned}
H & =\left(Q_{H}, D_{H}^{c}, N_{H}^{c}\right), \bar{H}=\left(\bar{Q}_{\bar{H}}, \bar{D}_{\bar{H}}^{c}, \bar{N}_{\bar{H}}^{c}\right), \\
h & =\left(D_{h}, D_{h}, D_{h}, H_{d}\right), \bar{h}=\left(\bar{D}_{\bar{h}}, \bar{D}_{\bar{h}}, \bar{D}_{\bar{h}}, H_{u}\right),
\end{aligned}
$$

where $H_{d}$ and $H_{u}$ are one pair of Higgs doublets in the MSSM. 
The $S U(5) \times U(1)_{X}$ gauge symmetry is broken down to the SM gauge symmetry by the following Higgs superpotential at the GUT scale

$$
W_{\mathrm{GUT}}=\lambda_{1} H H h+\lambda_{2} \overline{H H h}+\Phi\left(\bar{H} H-M_{\mathrm{H}}^{2}\right) .
$$

Only one F-flat and D-flat direction exists, which can be rotated along the $N_{H}^{c}$ and $\bar{N}_{H}^{c}$ directions. Hence, we obtain $<N_{H}^{c}>=<\bar{N}_{H}^{c}>=M_{\mathrm{H}}$. Furthermore, the superfields $H$ and $\bar{H}$ are "eaten" and acquire substantial masses via the supersymmetric Higgs mechanism, with the exception of $D_{H}^{c}$ and $\bar{D}_{\bar{H}}^{c}$. Additionally, the superpotential terms $\lambda_{1} H \mathrm{Hh}$ and $\lambda_{2} \overline{H H h}$ couple $D_{H}^{c}$ and $\bar{D} \frac{c}{H}$ respectively with $D_{h}$ and $\bar{D}_{\bar{h}}$, forming massive eigenstates with masses $2 \lambda_{1}<N_{H}^{c}>$ and $2 \lambda_{2}<\bar{N} \frac{c}{H}>$. As a result, we naturally experience the doublet-triplet splitting due to the missing partner mechanism [7]. The triplets in $h$ and $\bar{h}$ though only have a small mixing through the $\mu$ term, and as such, the colored Higgsinoexchange mediated proton decay is negligible, i.e., there is no dimension-5 proton decay problem.

To realize string-scale gauge coupling unification [8-10], we introduce the following vector-like particles (flippons) at the $\mathrm{TeV}$ scale

$$
\begin{aligned}
& X F=(\mathbf{1 0}, \mathbf{1}), \overline{X F}=(\overline{\mathbf{1 0}},-\mathbf{1}), \\
& X l=(\mathbf{1},-\mathbf{5}), \overline{X l}=(\mathbf{1}, \mathbf{5}) .
\end{aligned}
$$

The particle content from the decompositions of $X F, \overline{X F}$, $X l$, and $\overline{X l}$ under the SM gauge symmetry are

$$
\begin{aligned}
& X F=\left(X Q, X D^{c}, X N^{c}\right), \overline{X F}=\left(X Q^{c}, X D, X N\right), \\
& X l=X E, \overline{X l}=X E^{c} .
\end{aligned}
$$

The quantum numbers for the extra vector-like particles under the $S U(3)_{C} \times S U(2)_{L} \times U(1)_{Y}$ gauge symmetry are

$$
\begin{aligned}
& X Q=\left(\mathbf{3}, \mathbf{2}, \frac{\mathbf{1}}{\mathbf{6}}\right), X Q^{c}=\left(\overline{\mathbf{3}}, 2,-\frac{1}{6}\right), \\
& X D=\left(\mathbf{3}, \mathbf{1},-\frac{\mathbf{1}}{\mathbf{3}}\right), X D^{c}=\left(\overline{\mathbf{3}}, 1, \frac{1}{3}\right), \\
& X N=(\mathbf{1}, \mathbf{1}, \mathbf{0}), X N^{c}=(\mathbf{1}, \mathbf{1}, \mathbf{0}), \\
& X E=(\mathbf{1}, \mathbf{1},-\mathbf{1}), X E^{c}=(\mathbf{1}, \mathbf{1}, \mathbf{1}) .
\end{aligned}
$$

The superpotential is

$$
\begin{aligned}
W_{\text {Yukawa }}= & y_{i j}^{D} F_{i} F_{j} h+y_{i j}^{U v} F_{i} \bar{f}_{j} \bar{h}+y_{i j}^{E} \bar{l}_{i} \bar{f}_{j} h \\
& +\mu h \bar{h}+y_{i j}^{N} \phi_{i} \bar{H} F_{j}+M_{i j}^{\phi} \phi_{i} \phi_{j} \\
& +y_{X F} X F X F h+y_{\overline{X F}} \overline{X F X F h} \\
& +M_{X F} \overline{X F} X F+M_{X l} \overline{X l} X l
\end{aligned}
$$

and after the $S U(5) \times U(1)_{X}$ gauge symmetry is broken down to the SM gauge symmetry, the above superpotential gives

$$
\begin{aligned}
W_{S S M}= & y_{i j}^{D} D_{i}^{c} Q_{j} H_{d}+y_{j i}^{U v} U_{i}^{c} Q_{j} H_{u}+y_{i j}^{E} E_{i}^{c} L_{j} H_{d} \\
& +y_{i j}^{U v} N_{i}^{c} L_{j} H_{u}+\mu H_{d} H_{u}+y_{i j}^{N}\left\langle\bar{N} \bar{H}_{H}^{c}\right\rangle \phi_{i} N_{j}^{c}
\end{aligned}
$$

$$
\begin{aligned}
& +y_{X F} X Q X D^{c} H_{d}+y_{\overline{X F}} X Q^{c} X D H_{u} \\
& +M_{X F}\left(X Q^{c} X Q+X D^{c} X D\right) \\
& +M_{X l} X E^{c} X E+M_{i j}^{\phi} \phi_{i} \phi_{j} \\
& +\cdots\left(\text { decoupled below } M_{G U T}\right) .
\end{aligned}
$$

where $y_{i j}^{D}, y_{i j}^{U v}, y_{i j}^{E}, y_{i j}^{N}, y_{X F}$, and $y_{\overline{X F}}$ are Yukawa couplings, $\mu$ is the bilinear Higgs mass term, and $M_{i j}^{\phi}, M_{X F}$ and $M_{X l}$ are masses for new particles. These new particles are of course our flippons, however, we shall not explicitly compute the masses $M_{i j}^{\phi}, M_{X F}$, and $M_{X l}$ here in this work, reserving that project for a future date. Nonetheless, we do implement a common mass decoupling scale $M_{V}$ for the flippon vectorlike particles. Current LHC constraints on vector-like $T$ and $B$ quarks [28] provide lower limits of around $855 \mathrm{GeV}$ for our $\left(X Q, X Q^{c}\right)$ vector-like flippons and $735 \mathrm{GeV}$ for our $\left(X D, X D^{c}\right)$ vector-like flippons. Accordingly, we establish our lower $M_{V}$ limit at $M_{V} \geq 855 \mathrm{GeV}$ to ensure complete coverage of all experimentally viable flippon masses in our analysis.

As summarized in the prior section, the split-unification of flipped $S U$ (5) [5-7] provides for fundamental GUT scale Higgs representations (not adjoints), natural doublet-triplet splitting, suppression of dimension-five proton decay [29], and a two-step see-saw mechanism for neutrino masses [30,31]. Additions to the one-loop gauge $\beta$-function coefficients $b_{i}$ to include contributions from the vector-like flippon multiplets induce the necessary flattening of the $S U(3)$ Renormalization Group Equation (RGE) running $\left(b_{3}=0\right)$ [17], translating into a clear separation between the primary $S U(3)_{C} \times S U(2)_{L}$ unification near $10^{16} \mathrm{GeV}$ and the secondary $S U(5) \times U(1)_{X}$ unification at around $5 \times 10^{17} \mathrm{GeV}$, which we define as the $M_{\mathcal{F}}$ scale, thus elevating unification to near the Planck mass. At the primary $S U(3)_{C} \times S U(2)_{L}$ unification near $10^{16} \mathrm{GeV}$, the $M 2$ and $M 3$ gaugino mass terms are unified into a single term that we refer to as $M 5$ [32], where $M 5=M 2=M 3$ between the primary unification around $10^{16} \mathrm{GeV}$ and the secondary $S U(5) \times U(1)_{X}$ unification near $5 \times 10^{17} \mathrm{GeV}$ [17]. The $M 1$ gaugino mass term runs up to the secondary $S U(5) \times U(1)_{X}$ unification at $M_{\mathcal{F}}$ and unifies with $M 5$, by means of a slight shift due to $U(1)_{X}$ flux effects [32] between the primary unification around $10^{16} \mathrm{GeV}$ and the secondary $S U(5) \times U(1)_{X}$ unification at $M_{\mathcal{F}}$ [17]. Given this shift, we refer to the $M 1$ gaugino mass term above the primary unification around $10^{16} \mathrm{GeV}$ as $M_{1 X}$ [32]. The resulting baseline extension for logarithmic running of the No-Scale boundary conditions permits sufficient scale for natural dynamic evolution down to viable phenomenology at the electroweak scale. It is this associated flattening of the color-charged gaugino mass scale that produces the distinctive mass texture of $M\left(\tilde{t}_{1}\right)<M(\widetilde{g})<M(\widetilde{q})$, generating a light stop and gluino that are lighter than all other squarks [12]. 
Therefore, the most general supersymmetry breaking soft terms at the scale $M_{\mathcal{F}}$ in our $\mathcal{F}-S U(5)$ model are $M_{5}, M_{1 X}$, $M_{U^{c} L}, M_{E^{c}}, M_{Q D^{c} N^{c}}, M_{H_{u}}, M_{H_{d}}, A_{\tau}, A_{t}$, and $A_{b}$. General supersymmetry breaking soft terms of these type are partially inspired by D-brane model building [26], where $F_{i}$, $\bar{f}_{i}, \bar{l}_{i}$, and $h / \bar{h}$ arise from intersections of different stacks of D-branes. Consequently, the corresponding supersymmetry breaking soft mass terms and trilinear $A$ terms will be different, while $M_{H_{u}}$ is equal to $M_{H_{d}}$. Even though the Yukawa terms $H H h$ and $\overline{H H h}$ of Eq. (8) and $F_{i} F_{j} h, X F X F h$, and $\overline{X F X F h}$ of Eq. (15) are forbidden by the anomalous global $U(1)$ symmetry of $U(5)$, we might generate these Yukawa terms from high-dimensional operators or instanton effects. Unlike the $S U(5)$ models, the Yukawa term $F_{i} F_{j} h$ in the $\mathcal{F}-S U(5)$ model gives down-type quark masses, so their Yukawa couplings can be small and can be generated via high-dimensional operators or instanton effects.

\section{Numerical methodology}

The general SUSY breaking soft terms $M_{5}, M_{1 X}, M_{U^{c} L}$, $M_{E^{c}}, M_{Q D^{c} N^{c}}, M_{H_{u}}=M_{H_{d}}=M_{H}, A_{\tau}, A_{t}$, and $A_{b}$ are applied at the $M_{\mathcal{F}}$ scale near $M_{\mathcal{F}} \simeq 5 \times 10^{17} \mathrm{GeV}$ (in contrast to the traditional GUT scale of about $10^{16} \mathrm{GeV}$ in the MSSM), in addition to $\tan \beta$, the vector-like flippon mass decoupling scale $M_{V}$, and the top quark mass $M_{t}$. The parameter space is sampled within the limits $100 \leq M_{5} \leq 5000 \mathrm{GeV}, 100 \leq M_{1 X} \leq 5000 \mathrm{GeV}$, $100 \leq M_{U^{c} L} \leq 5000 \mathrm{GeV}, 100 \leq M_{E^{c}} \leq 5000 \mathrm{GeV}$, $100 \leq M_{Q D^{c} N^{c}} \leq 5000 \mathrm{GeV},-5000 \leq A_{\tau} \leq 5000 \mathrm{GeV}$, $-5000 \leq A_{t} \leq 5000 \mathrm{GeV},-5000 \leq A_{b} \leq 5000 \mathrm{GeV}$, $2 \leq \tan \beta \leq 60$, and $855 \leq M_{V} \leq 20,000 \mathrm{GeV}$. A rea- sonable tolerance of the top quark mass is permitted around the world average [33], employing upper and lower limits of $171.8 \leq M_{t} \leq 174.8 \mathrm{GeV}$. The WMAP 9-year [34] and 2015 Planck [35] relic density measurements are implemented, such that we constrain the model to satisfy both sets of data and allow the inclusion of multi-component dark matter beyond the neutralino, imposing limits of $\Omega h^{2} \leq 0.1221$. We will further identify the subset of points that are consistent with the recently released 2018 Planck observation [36] of $\Omega h^{2}=0.120 \pm 0.001$. Regarding LHC gluino searches, a firm lower limit is imposed on the gluino mass of $M(\widetilde{g}) \geq 1.6 \mathrm{TeV}$, allowing for a reasonable lower boundary under all the LHC gluino searches.

The light Higgs boson mass theoretical calculation is allowed to float around the experimental central value of $m_{h}=125.09 \mathrm{GeV}$, though we do enforce a larger range of $123 \leq m_{h} \leq 128 \mathrm{GeV}$ to account for a $2 \sigma$ experimental uncertainty over and above a theoretical uncertainty of $1.5 \mathrm{GeV}$ in our computations. The actual numerical value of the flippon Yukawa coupling remains an unknown, hence we permit the Yukawa coupling to range from a minimum value to its maximum in our light Higgs boson mass computations. A minimal flippon Yukawa coupling does not allow any vector-like flippon contributions, as the light Higgs boson mass in this case is then comprised of only the 1loop and 2-loop SUSY contributions, chiefly from the coupling to the light stop. A maximum flippon Yukawa coupling implies the $\left(X D, X D^{c}\right)$ flippon Yukawa coupling is fixed at $Y_{X D}=0$ and the $\left(X U, X U^{c}\right)$ flippon Yukawa coupling is set at $Y_{X U}=1$, with the $\left(X D, X D^{c}\right)$ flippon trilinear coupling $A$ term set at $A_{X D}=0$ and the $\left(X U, X U^{c}\right) A$ term fixed at $A_{X U}=A_{U}=A_{0}[12,13]$. When necessary, we shall choose the maximum flippon Yukawa coupling in

Table 1 The $\mathcal{F}-S U(5)$ general SUSY breaking soft terms, in addition to the vector-like flippon decoupling scale $M_{V}$, the low energy ratio of Higgs vacuum expectation values (VEVs) $\tan \beta$, and top quark mass

$M_{t}$, for the four regions of the model space we study in this work. Each benchmark point is identified with an alphabetical label in order to link the data in Table 1 with the data in Tables 2, 3. All masses are in GeV

\begin{tabular}{|c|c|c|c|c|c|c|c|c|c|c|c|c|c|}
\hline Model & benchmark & $M_{5}$ & $M_{1 \mathrm{X}}$ & $M_{U^{c} L}$ & $M_{E^{c}}$ & $M_{Q D^{c} N^{c}}$ & $M_{H}$ & $A_{\tau}$ & $A_{t}$ & $A_{b}$ & $M_{V}$ & $\tan \beta$ & $M_{t}$ \\
\hline Stop coannihilation & A & 1650 & 3125 & 2142 & 1158 & 3125 & 175 & 3125 & -4850 & 1250 & 16250 & 17.58 & 174.43 \\
\hline Stop coannihilation & $\mathrm{B}$ & 1800 & 3275 & 325 & 3275 & 3275 & 1308 & 325 & -4550 & -1500 & 16550 & 42.74 & 171.85 \\
\hline Stop coannihilation & $\mathrm{C}$ & 2325 & 3800 & 850 & 2817 & 2325 & 3800 & 2600 & -3500 & 2600 & 17600 & 24.33 & 174.56 \\
\hline Pure higgsino & $\mathrm{D}$ & 1700 & 4650 & 3667 & 3667 & 1700 & 2683 & 1250 & 4300 & -1800 & 4055 & 32.83 & 173.43 \\
\hline Pure higgsino & $\mathrm{E}$ & 2050 & 5000 & 3525 & 5000 & 5000 & 5000 & -1100 & 1950 & -1100 & 4755 & 48.16 & 174.80 \\
\hline Pure higgsino & $\mathrm{F}$ & 2375 & 3850 & 2867 & 3850 & 2375 & 3850 & -350 & 2700 & -350 & 2455 & 36.66 & 173.26 \\
\hline Higgs funnel & $\mathrm{G}$ & 1500 & 2975 & 4450 & 2483 & 2975 & 2483 & 550 & 3600 & -2500 & 3655 & 30.83 & 173.38 \\
\hline Higgs funnel & $\mathrm{H}$ & 1750 & 3225 & 3225 & 2242 & 275 & 1258 & 275 & 1450 & -1600 & 16450 & 30.41 & 173.14 \\
\hline Higgs funnel & I & 2300 & 3775 & 3775 & 2792 & 825 & 2792 & 3775 & 2550 & -500 & 9928 & 35.91 & 173.25 \\
\hline Stau coannihilation & $\mathrm{J}$ & 1600 & 1600 & 125 & 1108 & 1600 & 1108 & 125 & -1900 & 1150 & 16150 & 28.91 & 173.11 \\
\hline Stau coannihilation & $\mathrm{K}$ & 2175 & 3650 & 700 & 1683 & 700 & 2667 & -750 & 2300 & 2300 & 9678 & 46.49 & 171.92 \\
\hline Stau coannihilation & $\mathrm{L}$ & 2625 & 4100 & 1150 & 3117 & 2625 & 1150 & -2900 & -2900 & 3200 & 2955 & 50.99 & 172.01 \\
\hline
\end{tabular}


Table 2 Relevant SUSY spectrum masses for the $\mathcal{F}-S U(5)$ general SUSY breaking soft terms of Table 1. The soft SUSY breaking terms that generate each of these spectra can be identified by the alphabetical label. A $\dagger$ symbol in the light Higgs boson mass $m_{h}$ column represents the theoretically computed value consisting of only the 1-loop and 2- loop SUSY contributions, primarily from the coupling to the light stop, but does not include any vector-like flippon contributions, whereas a $\dagger$ symbol represents the 1-loop and 2-loop SUSY contributions plus the maximum vector-like flippon contribution. All masses are in $\mathrm{GeV}$

\begin{tabular}{|c|c|c|c|c|c|c|c|c|c|c|}
\hline Model & Benchmark & $M\left(\tilde{\chi}_{1}^{0}\right)$ & $M\left(\tilde{\chi}_{2}^{0}\right)$ & $M\left(\tilde{\chi}_{1}^{ \pm}\right)$ & $M\left(\tilde{\tau}_{1}^{ \pm}\right)$ & $M\left(\tilde{t}_{1}\right)$ & $M\left(\tilde{u}_{R}\right)$ & $M(\tilde{g})$ & $m_{h}$ & $M\left(H^{0} / A^{0}\right)$ \\
\hline Stop coannihilation & A & 693 & 781 & 781 & 1080 & 729 & 3759 & 2225 & $127.34^{\dagger}$ & 4484 \\
\hline Stop coannihilation & $\mathrm{B}$ & 728 & 850 & 850 & 2058 & 766 & 3727 & 2397 & $125.85^{\dagger}$ & 3927 \\
\hline Stop coannihilation & $\mathrm{C}$ & 862 & 1105 & 1105 & 2418 & 895 & 4370 & 3020 & $127.60^{\dagger}$ & 5134 \\
\hline Pure higgsino & $\mathrm{D}$ & 188 & -198 & 192 & 3729 & 3012 & 4695 & 2246 & $126.73^{\dagger \dagger}$ & 1268 \\
\hline Pure higgsino & $\mathrm{E}$ & 411 & -421 & 415 & 3241 & 3586 & 5591 & 2726 & $126.21^{\dagger}$ & 1794 \\
\hline Pure higgsino & $\mathrm{F}$ & 374 & -383 & 378 & 3482 & 3699 & 5293 & 3068 & $125.99^{\dagger}$ & 2811 \\
\hline Higgs funnel & G & 621 & 679 & 679 & 3326 & 3556 & 4909 & 2028 & $127.18^{\dagger \dagger}$ & 1316 \\
\hline Higgs funnel & $\mathrm{H}$ & 717 & 824 & 824 & 3045 & 2729 & 4172 & 2337 & $124.87^{\dagger \dagger}$ & 1449 \\
\hline Higgs funnel & I & 840 & 1068 & 1068 & 3435 & 3564 & 5239 & 2989 & $124.67^{\dagger}$ & 1739 \\
\hline Stau coannihilation & $\mathrm{J}$ & 362 & 749 & 749 & 365 & 1248 & 2937 & 2121 & $126.77^{\dagger}$ & 3110 \\
\hline Stau coannihilation & $\mathrm{K}$ & 799 & 918 & 912 & 802 & 2729 & 3985 & 2805 & $125.62^{\dagger \dagger}$ & 1781 \\
\hline Stau coannihilation & $\mathrm{L}$ & 870 & 1177 & 1177 & 876 & 2632 & 5148 & 3278 & $126.43^{\dagger}$ & 4179 \\
\hline
\end{tabular}

Table 3 Relic density $\left(\Omega h^{2}\right)$, rare decay processes $\left(\Delta a_{\mu}, B r(b \rightarrow s \gamma)\right.$, $\operatorname{Br}\left(B_{s}^{0} \rightarrow \mu^{+} \mu^{-}\right)$, rescaled dark matter direct-detection cross sections $\left(\sigma_{S I}, \sigma_{S D}\right)$, and $p \rightarrow e^{+} \pi^{0}$ proton decay rates $\left(\tau_{p}\right)$ for the $\mathcal{F}-S U(5)$ general SUSY breaking soft terms of Table 1. The soft SUSY breaking terms that generate each of these values can be identified by the alphabetical label. The $\sigma_{S I}$ and $\sigma_{S D}$ cross-sections have been rescaled in accordance with Eq. (17). The numerical values given for $\Delta a_{\mu}$ are $\times 10^{-10}, \operatorname{Br}(b \rightarrow s \gamma)$ are $\times 10^{-4}, \operatorname{Br}\left(B_{s}^{0} \rightarrow \mu^{+} \mu^{-}\right)$are $\times 10^{-9}$, rescaled spin-independent cross-sections $\sigma_{S I}$ are $\times 10^{-11} \mathrm{pb}$, rescaled spin-dependent cross-sections $\sigma_{S D}$ are $\times 10^{-9} \mathrm{pb}$, and proton decay rate $\tau_{p}$ are $\times 10^{35} \mathrm{yrs}$

\begin{tabular}{llllllllll}
\hline Model & Benchmark & $\Omega h^{2}$ & $\Delta a_{\mu}$ & $\operatorname{Br}(b \rightarrow s \gamma)$ & $\operatorname{Br}\left(B_{s}^{0} \rightarrow \mu^{+} \mu^{-}\right)$ & $\sigma_{S I}$ & $\sigma_{S D}$ & $\tau_{p}$ & LSP \\
\hline Stop coannihilation & $\mathrm{A}$ & 0.1115 & 0.40 & 3.56 & 3.16 & 0.1 & 0.1 & 3.9 & $>99 \%$ Bino \\
Stop coannihilation & $\mathrm{B}$ & 0.1219 & 1.02 & 3.45 & 3.57 & 0.1 & 0.1 & 5.9 & $>99 \%$ Bino \\
Stop coannihilation & $\mathrm{C}$ & 0.1045 & 0.60 & 3.47 & 3.20 & 0.6 & 1.0 & 2.7 & $>99 \%$ Bino \\
Pure higgsino & $\mathrm{D}$ & 0.0052 & 0.74 & 3.72 & 2.90 & 34 & 1471 & 0.3 & $>99 \%$ Higgsino \\
Pure higgsino & $\mathrm{E}$ & 0.0198 & 0.99 & 3.64 & 3.08 & 125 & 1484 & 0.8 & $>98 \%$ Higgsino \\
Pure higgsino & $\mathrm{F}$ & 0.0170 & 0.93 & 3.57 & 3.04 & 77 & 1305 & 0.4 & $>98 \%$ Higgsino \\
Higgs funnel & $\mathrm{G}$ & 0.1194 & 0.57 & 3.71 & 3.10 & 15 & 40.8 & 2.0 & $>99 \%$ Bino \\
Higgs funnel & $\mathrm{H}$ & 0.1193 & 0.76 & 3.61 & 3.38 & 6.4 & 13.2 & 2.6 & $>99 \%$ Bino \\
Higgs funnel & $\mathrm{I}$ & 0.1210 & 0.77 & 3.60 & 3.27 & 46 & 112 & 1.8 & $>99 \%$ Bino \\
Stau coannihilation & $\mathrm{J}$ & 0.1189 & 1.81 & 3.44 & 3.26 & 0.3 & 1.1 & 2.9 & $>99 \%$ Bino \\
Stau coannihilation & $\mathrm{K}$ & 0.1202 & 2.51 & 3.47 & 3.54 & 940 & 2746 & 1.3 & $>93 \%$ Bino \\
Stau coannihilation & $\mathrm{L}$ & 0.1176 & 0.79 & 3.49 & 3.65 & 0.1 & 0.1 & 4.4 & $>99 \%$ Bino \\
\hline
\end{tabular}

order to lift the light Higgs boson mass up to its observed value. However, some points in the model space can reach the Higgs boson observed value with no flippon contribution, thus we choose the minimal coupling in these instances. Regardless of our choice for the numerical value of the flippon Yukawa coupling, the calculation must return a value within $123 \leq m_{h} \leq 128 \mathrm{GeV}$. For our chosen benchmarks points that are meant to be sufficiently representative of the model space, we will clearly annotate as to whether the given light Higgs boson mass consists of the minimum or maximum flippon Yukawa coupling.
The model space is additionally constrained via rare decay, direct dark matter detection, and proton decay experimental results. The rare decay experimental constraints include the branching ratio of the rare b-quark decay of $\mathrm{Br}(b \rightarrow$ $s \gamma)=\left(3.43 \pm 0.21^{\text {stat }} \pm 0.24^{\text {th }} \pm 0.07^{\text {sys }}\right) \times 10^{-4}[37]$, the branching ratio of the rare B-meson decay to a dimuon of $\operatorname{Br}\left(B_{s}^{0} \rightarrow \mu^{+} \mu^{-}\right)=\left(2.9 \pm 0.7 \pm 0.29^{\text {th }}\right) \times 10^{-9}$ [38], and the $3 \sigma$ intervals around the Standard Model result and experimental measurement of the SUSY contribution to the anomalous magnetic moment of the muon of $-17.7 \times$ $10^{-10} \leq \Delta a_{\mu} \leq 43.8 \times 10^{-10}$ [39]. Direct dark matter 

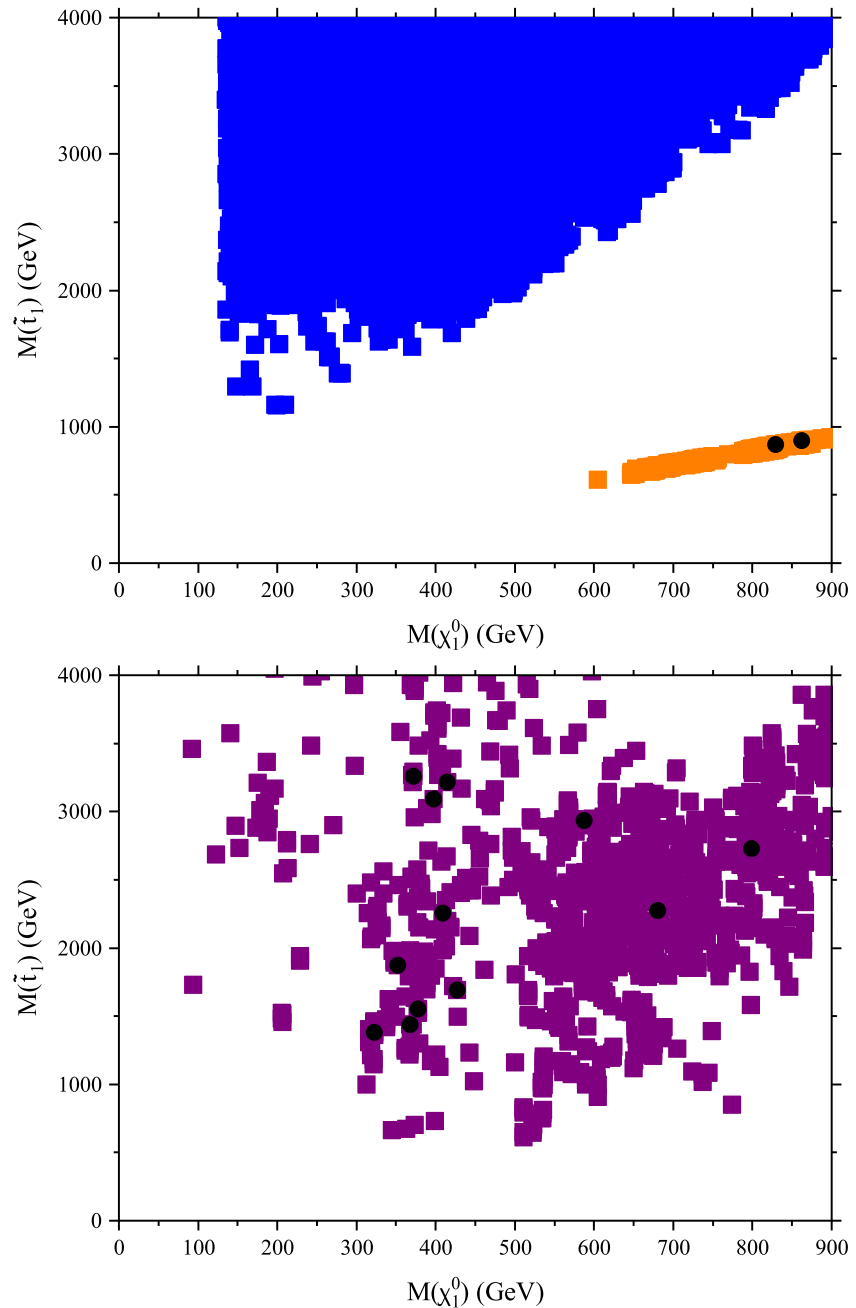

Fig. 1 Light stop mass $M\left(\widetilde{t}_{1}\right)$ as a function of the lightest neutralino mass $M\left(\widetilde{\chi}_{1}^{0}\right)$ for the four regions of the model space we study in this work. The stop coannihilation strip can be observed in the upper left plot space. Notice that there is no intersection between the Light Stop Coannihilation and pure Higgsino regions, though a more detailed analysis relaxing the higgsino and light stop coannihilation requirements we applied here is in progress [50] to ascertain any potential union

detection constraints encompass limits on spin-independent cross-sections for neutralino-nucleus interactions established by the Large Underground Xenon (LUX) experiment [40], PandaX-II Experiment [41], and XENON100 Collaboration [42], and limits on the proton spin-dependent cross-sections by the COUPP Collaboration [43] and XENON100 Collaboration [44]. Finally, we assess our $S U(5) \times U(1)_{X}$ grand unification against the current limits of about $1.7 \times 10^{34} \mathrm{yrs}$ on the proton decay rate $p \rightarrow e^{+} \pi^{0}[45]$.

A total of 110 million points were randomly sampled in scans implementing the $S U(5) \times U(1)_{X} M_{\mathcal{F}}$ scale boundary conditions, with only about 37,000 of those points surviving the applied constraints. The SUSY mass spectra, relic density, rare decay processes, and direct

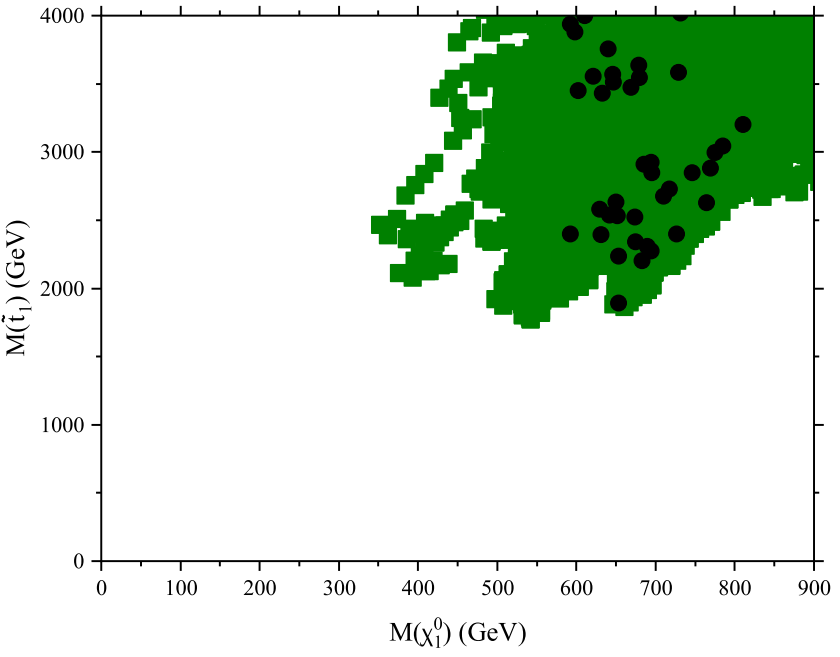

$\begin{array}{ll}\text { - } & \text { Stop Coannihilation (STOPC) } \\ \text { - } & \text { Higgsino LSP Funnel (HF) } \\ \text { - } & \text { Stau Coannihilation (STAUC) } \\ \text { - } & \Omega h^{2}=0.1200 \pm 0.001\end{array}$

of these two significant regions. All points plot satisfy the light Higgs boson mass and relic density observations we outlined in this work, in addition to rare decay and current LHC SUSY search constraints. The round black dots represent those points that can also satisfy the recent 2018 Planck Collaboration satellite relic density measurements of $\Omega h^{2}=0.120 \pm 0.001$

dark matter detection cross-sections are calculated with MicrOMEGAs 2.1 [46] utilizing a proprietary mpi modification of the SuSpect 2.34 [47] codebase to run flippon and General No-Scale $\mathcal{F}$-SU (5) enhanced RGEs, employing non-universal soft supersymmetry breaking parameters at the $M_{\mathcal{F}}$ scale. Supersymmetric particle decays are computed with SUSY-HIT 1.5a [48]. The Particle Data Group [49] world average for the strong coupling constant is $\alpha_{S}\left(M_{Z}\right)=$ $0.1181 \pm 0.0011$ at $1 \sigma$, and we assume a value in this work of $\alpha_{S}=0.1184$.

A total of 12 viable benchmark points are chosen to be reasonably representative of the model space for a given set of parameters $\left(M_{5}, M_{1 X}, M_{U^{c} L}, M_{E^{c}}, M_{Q D^{c} N^{c}}, M_{H}, A_{\tau}\right.$, $\left.A_{t}, A_{b}, M_{V}, \tan \beta, M_{t}\right)$. The data results are distributed across 

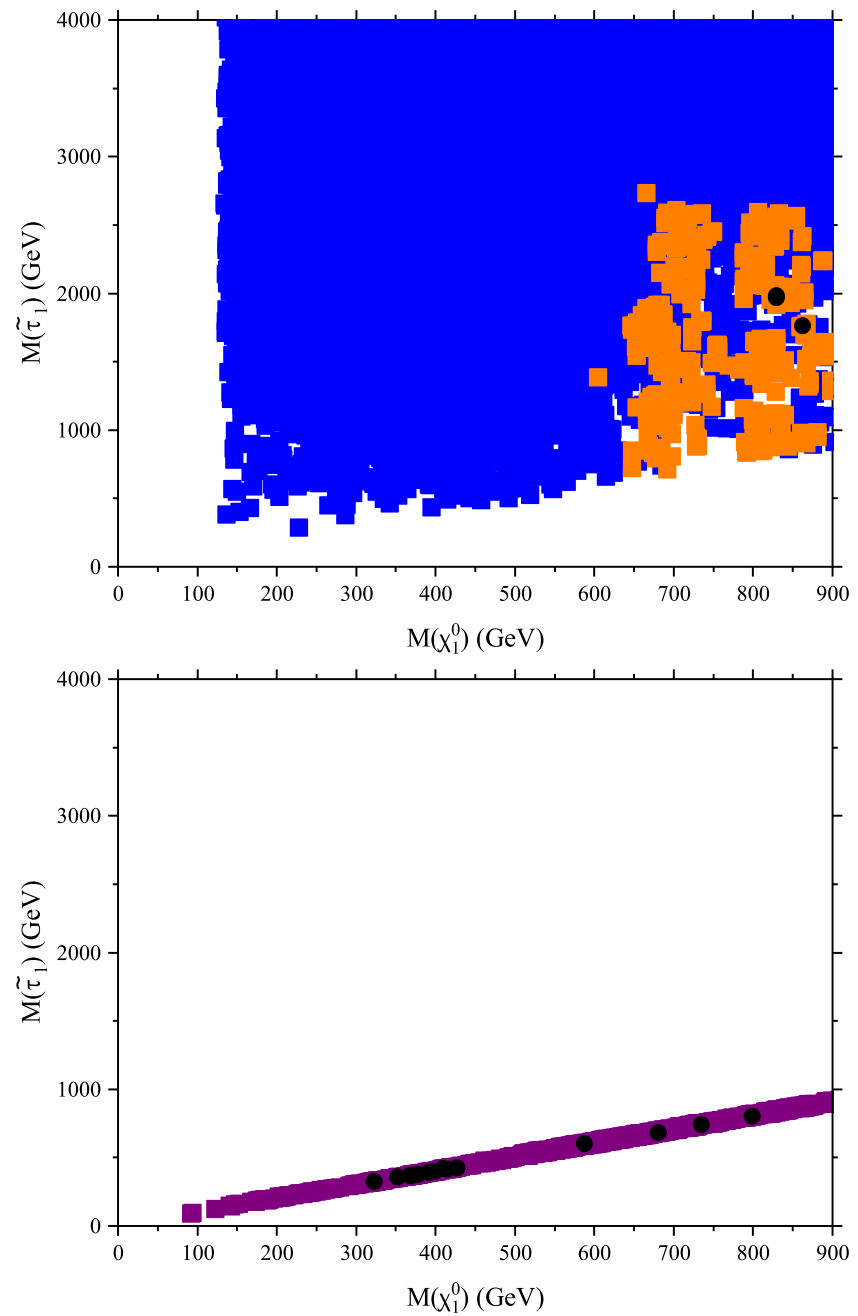

Fig. 2 Light stau mass $M\left(\widetilde{\tau}_{1}\right)$ as a function of the lightest neutralino mass $M\left(\widetilde{\chi}_{1}^{0}\right)$ for the four regions of the model space we study in this work. The stau coannihilation strip can be observed in the lower left plot space. All points plot satisfy the light Higgs boson mass and relic den-

Tables 1, 2 and 3. The benchmarks models of Tables 1, 2 and 3 originate via categorization of the viable model space into four dissimilar regions identified by LSP and NLSP characteristics, to be discussed shortly. The numerical relic density figures annotated in Table 3 consist only of the SUSY lightest neutralino $\tilde{\chi}_{1}^{0}$ abundance, therefore regions with numerical values less than the WMAP9 $1 \sigma$ measurement lower bound of about $\Omega h^{2} \simeq 0.1093$ must welcome alternative additions to the total observed relic density by WMAP9 and Planck. Given the potential for multi-component dark matter in regions of low neutralino density, the spin-dependent and spin-independent cross-section calculations provided in Table 3 are rescaled per the following expression:

$\sigma_{S I(S D)}^{\text {re-scaled }}=\sigma_{S I(S D)} \frac{\Omega h^{2}}{0.1138}$

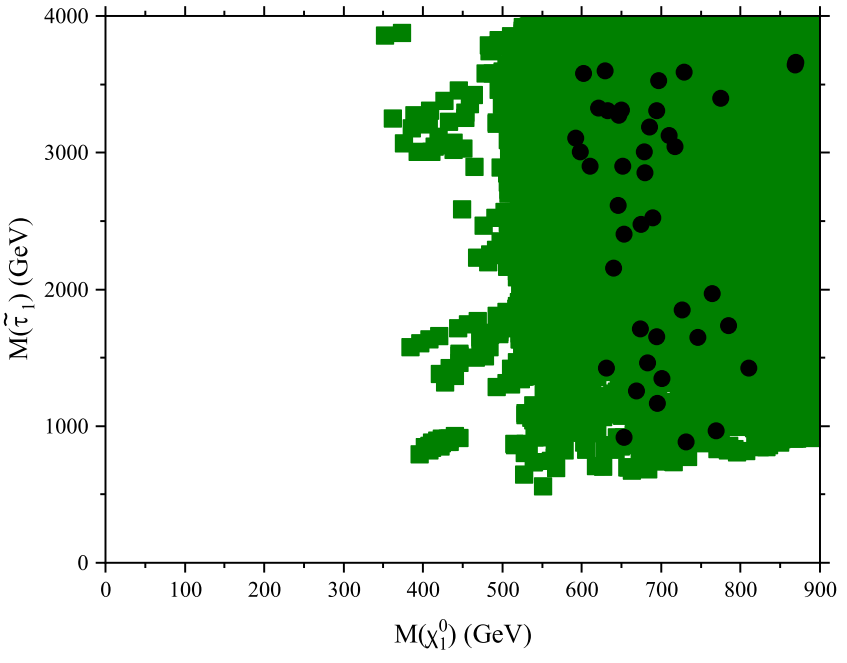

\begin{tabular}{|ll|}
\hline - & Stop Coannihilation (STOPC) \\
- & Higgsino LSP (HLSP) \\
- & Stau Coannel (HF) \\
- & $\Omega h^{2}=0.1200 \pm 0.001$ \\
\hline
\end{tabular}

sity observations we outlined in this work, in addition to rare decay and current LHC SUSY search constraints. The round black dots represent those points that can also satisfy the recent 2018 Planck Collaboration satellite relic density measurements of $\Omega h^{2}=0.120 \pm 0.001$

\section{Phenomenological results}

The model space is constrained per the experimental constraints detailed in the prior section, with the exception of the LUX, PandaX-II, and XENON cross-sections, which we shall evaluate independently of all the other empirical measurements. Post application of the constraints, the surviving viable parameter space consists of four interesting regions segregated by LSP characteristics and the NLSP. The four regions are identified as (1) light stop coannihilation (STOPC), with $M\left(\widetilde{t}_{1}\right)-M\left(\widetilde{\chi}_{1}^{0}\right) \leq 50 \mathrm{GeV}$; (2) pure higgsino LSP (HLSP), restricted to the conditions $M\left(\widetilde{\chi}_{2}^{0}\right)-M\left(\widetilde{\chi}_{1}^{0}\right)=9-10 \mathrm{GeV}, M\left(\widetilde{\chi}_{1}^{ \pm}\right)-M\left(\tilde{\chi}_{1}^{0}\right)=$ $3-7 \mathrm{GeV}, M\left(\widetilde{\chi}_{2}^{0}\right)<0$, which consistently generates an LSP greater than 98\% higgsino; (3) Higgs Funnel (HF), with $M\left(H^{0} / A^{0}\right) \sim 2 M\left(\widetilde{\chi}_{1}^{0}\right)$, where we allow a tolerance of 

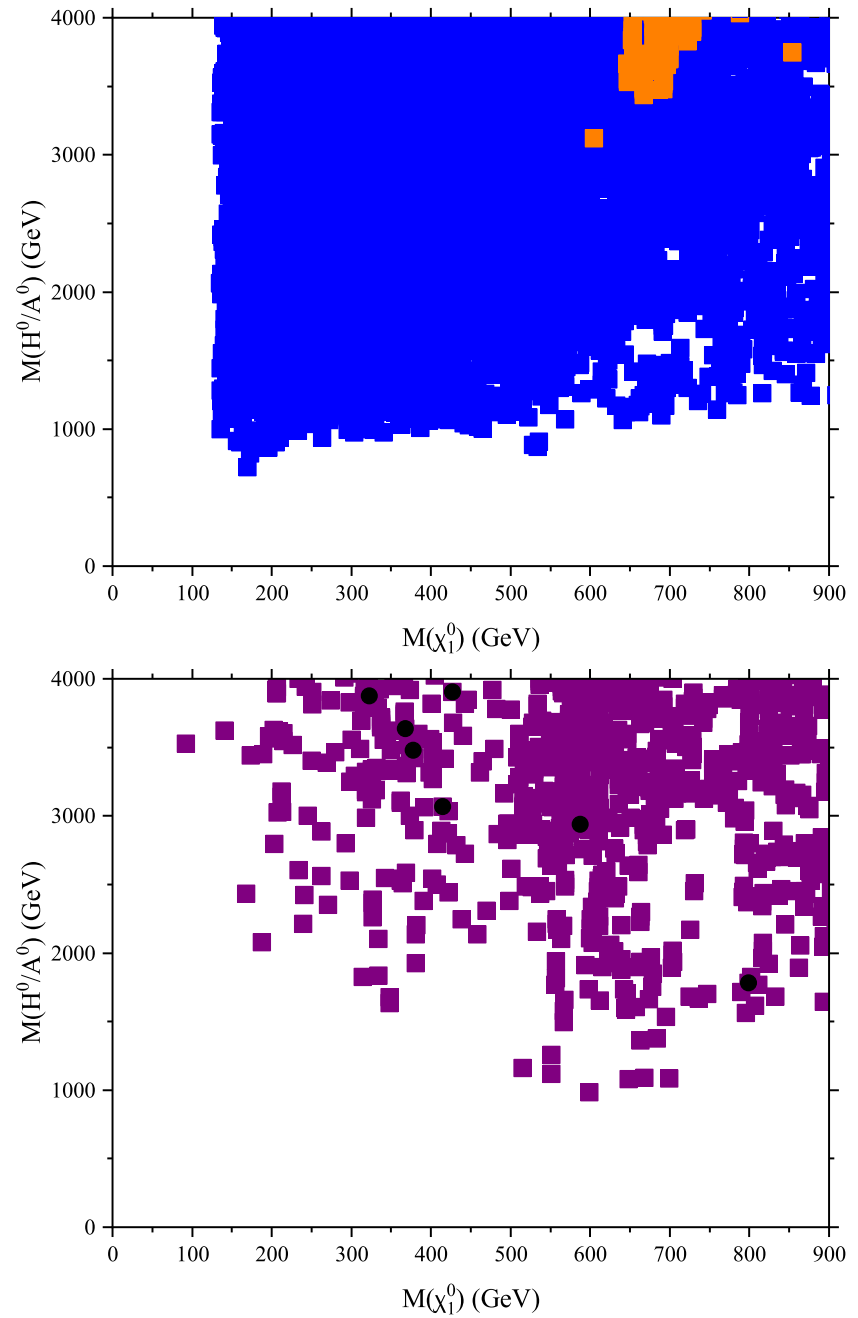

Fig. 3 Heavy neutral and pseudoscalar SUSY Higgs mass $M\left(H^{0} / A^{0}\right)$ as a function of the lightest neutralino mass $M\left(\widetilde{\chi}_{1}^{0}\right)$ for the four regions of the model space we study in this work. The Higgs Funnel region can be observed in the upper right plot space. All points plot satisfy the light Higgs boson mass and relic density observations we outlined

$\pm 100 \mathrm{GeV}$ on $M\left(H^{0} / A^{0}\right)$; and (4) light stau coannihilation (STAUC), with $M\left(\widetilde{\tau}_{1}^{ \pm}\right)-M\left(\widetilde{\chi}_{1}^{0}\right) \leq 20 \mathrm{GeV}$. The light stop coannihilation, Higgs Funnel, and light stau coannihilation regions all generate an LSP that is almost entirely bino. The lower mass boundaries of all these four regions are essentially defined by the LHC constraints, which include the gluino and other ATLAS and CMS search constraints we shall discuss in the forthcoming paragraphs.

The light stop $\left(\widetilde{t}_{1}\right)$ is the NLSP for all of the stop coannihilation points. The very narrow 2018 Planck relic density of $\Omega h^{2}=0.120 \pm 0.001$ can be generated with $M\left(\widetilde{t}_{1}\right)-M\left(\widetilde{\chi}_{1}^{0}\right) \simeq 35-40 \mathrm{GeV}$. The STOPC region is illustrated in Figs. 1, 2, 3 and 4, with Fig. 1 displaying the light stop mass as a function of the LSP $\widetilde{\chi}_{1}^{0}$, clearly indicating the light stop coannihilation strip, with the lower end severed by

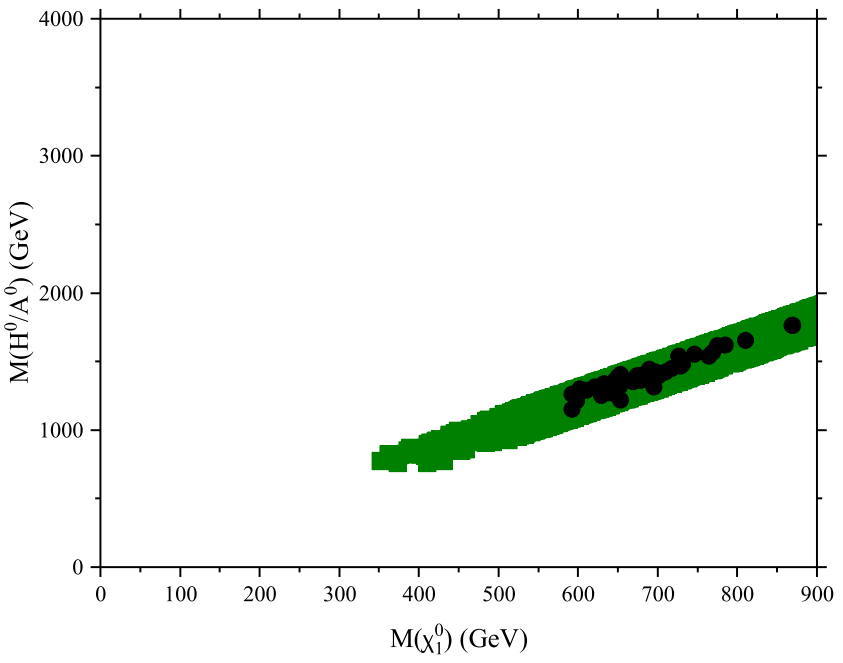

$\begin{array}{ll}\text { - } & \text { Stop Coannihilation (STOPC) } \\ \text { - } & \text { Higgsino LSP (HLSP) } \\ \text { - } & \text { Stags Funnel (HF) } \\ \text { - } & \Omega h^{2}=0.1200 \pm 0.001\end{array}$

in this work, in addition to rare decay and current LHC SUSY search constraints. The round black dots represent those points that can also satisfy the recent 2018 Planck Collaboration satellite relic density measurements of $\Omega h^{2}=0.120 \pm 0.001$

the most recent LHC constraints. Given that the small mass delta we require is less than $M_{t}$, the light stop is forced into the decay channel $\widetilde{t}_{1} \rightarrow c+\widetilde{\chi}_{1}^{0}$ and the gluino will always produce a light stop $\left(\widetilde{g} \rightarrow \widetilde{t}_{1} t\right)$, as highlighted in Table 4 . The near degenerate LSP and light stop in this STOPC channel can evade the current ATLAS [51,52] and CMS [53-56] LHC light stop searches. Applying the strongest constraints from both ATLAS and CMS on $M\left(\tilde{t}_{1}\right)-M\left(\widetilde{\chi}_{1}^{0}\right)$ and $M\left(\widetilde{t}_{1}\right)$ requires us to enforce $M\left(\widetilde{t}_{1}\right)>550 \mathrm{GeV}$ for our given mass delta of $M\left(\widetilde{t}_{1}\right)-M\left(\widetilde{\chi}_{1}^{0}\right) \leq 50 \mathrm{GeV}$, defining the lower end of the light stop coannihilation strip in Fig. 1. This constraint is included in all of Figs. 1, 2, 3 and 4.

The higgsino spectra are identified by the Higgs bilinear mass term $\mu$ driven via RGE running below the gaugino mass terms $M_{1}$ and $M_{2}$ at low-energy near electroweak symmetry 

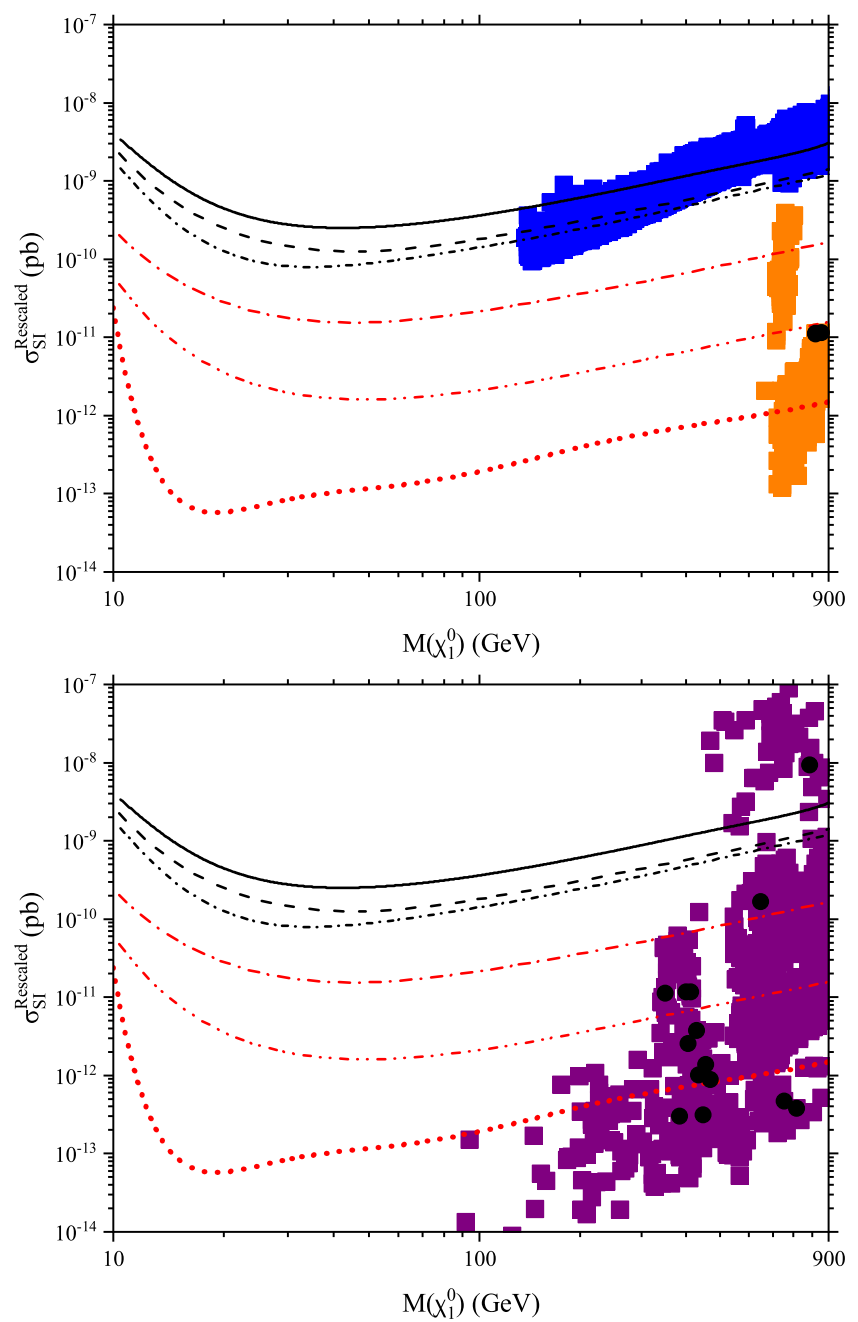

Fig. 4 Illustration of the PandaX-II, LUX, and XENON WIMPnucleon spin-independent cross-section constraints applied to the model space studied in this work. The $\sigma_{S I}$ cross-sections have been rescaled in accordance with Eq. (17). All points plot satisfy the light Higgs boson mass and relic density observations we outlined in this work,

breaking (EWSB), thus driving the $\widetilde{\chi}_{2}^{0}$ mass negative. This is despite the fact that the $\mu$ term can be larger or smaller than the gaugino mass terms $M_{5}$ and $M_{1 X}$ at the $M_{\mathcal{F}}$ scale. The tight constraints applied within the higgsino region ensure an almost $100 \%$ higgsino LSP, though it apparently does prevent any combination of a pure higgsino LSP and light stop NLSP. This is evident in Fig. 1 by observing the gap between the HLSP and STOPC points, as no spectra with $M\left(\widetilde{(}_{1}\right)-M\left(\widetilde{\chi}_{1}^{0}\right) \leq 50 \mathrm{GeV}$ can also generate a pure higgsino LSP. This notwithstanding, preliminary studies do show that a STOPC point with $M\left(\widetilde{t}_{1}\right)-M\left(\widetilde{\chi}_{1}^{0}\right) \leq 50 \mathrm{GeV}$ can possess an LSP that is dominantly higgsino, for example, more than $60 \%$ and potentially as high as $80 \%$ or greater. While we do not integrate this more detailed HLSP+STOPC analysis into this work, it is currently in progress [50]. Such a combination
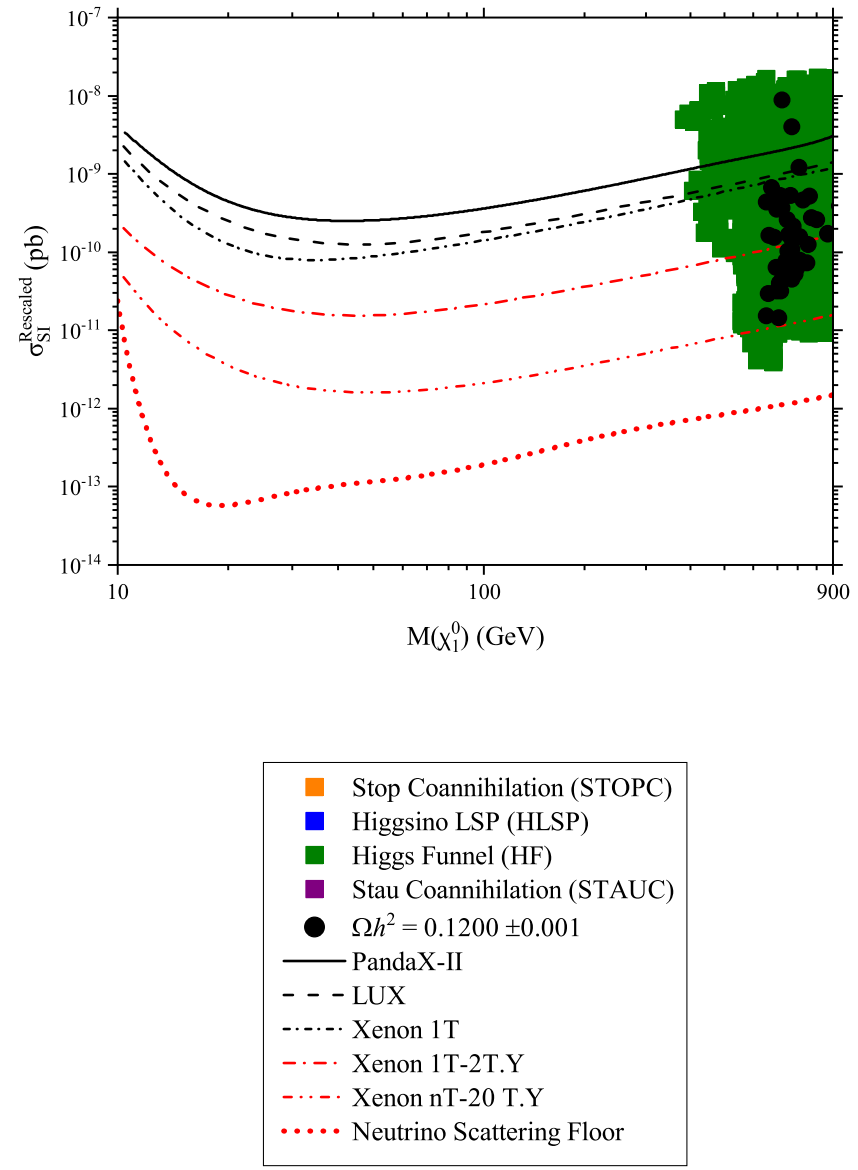

in addition to rare decay and current LHC SUSY search constraints. The round black dots represent those points that can also satisfy the recent 2018 Planck Collaboration satellite relic density measurements of $\Omega h^{2}=0.120 \pm 0.001$

of a dominantly higgsino LSP and light stop NLSP is rather difficult to produce naturally [57], though it has been uncovered in certain realistic intersecting D6-brane models [58]. As a result of our tight constraints on the higgsino in order to generate a pure higgsino LSP, most of the higgsino points in Figs. 1, 2, 3 and 4 have $M\left(\widetilde{t}_{1}\right)>M(\widetilde{g})$, thus we focus on these primary higgsino spectra here in this work. Unfortunately, as depicted in Table 4, there is no dominant decay channel to consistent final states for either the light stop or gluino in the HLSP region. As can be inferred from Table 4 and our constraint of $M\left(\tilde{\chi}_{1}^{ \pm}\right)-M\left(\tilde{\chi}_{1}^{0}\right)=3-7 \mathrm{GeV}$, the chargino participates in the off-shell Standard Model boson mediated decays $\tilde{\chi}_{1}^{ \pm} \rightarrow u \bar{d} / c \bar{s}+\widetilde{\chi}_{1}^{0}(36 \% / 36 \%)$ or $\tilde{\chi}_{1}^{ \pm} \rightarrow l^{ \pm}+v_{l}+\widetilde{\chi}_{1}^{0}$ (28\%), and these pure higgsino points have an LHC estab- 
Table 4 Dominant gluino $(\widetilde{g})$ and light stop $\left(\widetilde{t_{1}}\right)$ decay modes for each of the regions of the model space we study in this work, along with the associated branching ratios. Here, $q=(u, d, c, s)$ and $\widetilde{q}=(\widetilde{u}, \widetilde{d}, \widetilde{c}, \widetilde{s})$

\begin{tabular}{|c|c|c|}
\hline Model & Dominant decay mode & Branching ratio \\
\hline \multirow[t]{2}{*}{ Stop coannihilation } & $\widetilde{g} \rightarrow \widetilde{t}_{1} t$ & $100 \%$ \\
\hline & $\tilde{t}_{1} \rightarrow c+\tilde{\chi}_{1}^{0}$ & $80-98 \%$ \\
\hline \multirow[t]{4}{*}{ Pure higgsino } & $\tilde{g} \rightarrow \tilde{\chi}_{1}^{ \pm}+t+b \rightarrow q \bar{q}+t+b+\tilde{\chi}_{1}^{0}$ & $\sim 28 \%$ \\
\hline & $\widetilde{g} \rightarrow t \bar{t}+\tilde{\chi}_{1}^{0}$ & $\sim 14 \%$ \\
\hline & $\widetilde{t}_{1} \rightarrow \widetilde{g}+t$ & $\sim 32 \%$ \\
\hline & $\tilde{t}_{1} \rightarrow \tilde{\chi}_{1}^{ \pm}+b \rightarrow q \bar{q}+b+\tilde{\chi}_{1}^{0}$ & $\sim 16 \%$ \\
\hline \multirow[t]{7}{*}{ Higgs funnel } & $\tilde{g} \rightarrow \tilde{\chi}_{1}^{ \pm}+t+b \rightarrow q \bar{q}+t+b+\tilde{\chi}_{1}^{0}\left[M\left(\tilde{\chi}_{1}^{ \pm}\right)-M\left(\tilde{\chi}_{1}^{0}\right)<M\left(W^{ \pm}\right)\right]$ & $\sim 21 \%$ \\
\hline & $\begin{array}{l}\widetilde{g} \rightarrow \widetilde{\chi}_{1}^{ \pm}+t+b \rightarrow W^{ \pm}+t+b+\widetilde{\chi}_{1}^{0}\left[M\left(\tilde{\chi}_{1}^{ \pm}\right)-M\left(\widetilde{\chi}_{1}^{0}\right)>M\left(W^{ \pm}\right)\right] \\
\text {Larger } M\left(\widetilde{t}_{1}\right)-M(\widetilde{g})\end{array}$ & $\sim 26 \%$ \\
\hline & $\widetilde{t}_{1} \rightarrow \widetilde{g}+t$ & $\sim 48 \%$ \\
\hline & $\tilde{t}_{1} \rightarrow \tilde{\chi}_{1}^{ \pm}+b \rightarrow q \bar{q}+b+\tilde{\chi}_{1}^{0}$ & $\sim 15 \%$ \\
\hline & $\underline{\text { Smaller } M\left(\widetilde{t}_{1}\right)-M(\widetilde{g})}$ & \\
\hline & $\tilde{t}_{1} \rightarrow \widetilde{g}+t$ & $\sim 18 \%$ \\
\hline & $\tilde{t}_{1} \rightarrow \tilde{\chi}_{1}^{ \pm}+b \rightarrow W^{ \pm}+b+\tilde{\chi}_{1}^{0}$ & $\sim 39 \%$ \\
\hline \multirow[t]{6}{*}{ Stau coannihilation } & $\underline{M(\widetilde{g})-M\left(\widetilde{t_{1}}\right)>M_{t}}$ & \\
\hline & $\widetilde{g} \rightarrow \widetilde{t}_{1} t \rightarrow t \bar{t}+\tilde{\chi}_{1}^{0}$ & $\sim 100 \%$ \\
\hline & $\tilde{t}_{1} \rightarrow t+\tilde{\chi}_{1}^{0}$ & $\sim 100 \%$ \\
\hline & $M(\widetilde{g})-M\left(\widetilde{t}_{1}\right)<M_{t}$ & \\
\hline & $\tilde{g} \rightarrow t \bar{t}+\tilde{\chi}_{1}^{0}$ & $\sim 6 \%$ \\
\hline & $\tilde{t}_{1} \rightarrow \tilde{\chi}_{1}^{ \pm}+b \rightarrow \tilde{\tau}_{1}^{ \pm}+v_{\tau}+b \rightarrow \tau^{ \pm}+v_{\tau}+b+\tilde{\chi}_{1}^{0}$ & $\sim 18 \%$ \\
\hline
\end{tabular}

lished lower bound of about $M\left(\tilde{\chi}_{1}^{ \pm}\right) \sim 140 \mathrm{GeV}$ [59], thus we enforce $M\left(\tilde{\chi}_{1}^{ \pm}\right)>140 \mathrm{GeV}$ in Figs. 1, 2, 3 and 4 .

The chargino $\tilde{\chi}_{1}^{ \pm}$is the NLSP for $99.6 \%$ of the Higgs Funnel points, with the remaining $0.4 \%$ possessing a stau NLSP. Nonetheless, only $0.07 \%$ of the Higgs Funnel also resides in the $M\left(\tilde{\tau}_{1}^{ \pm}\right)-M\left(\tilde{\chi}_{1}^{0}\right) \leq 20 \mathrm{GeV}$ stau coannihilation strip. We further observe that $11.5 \%$ of the Higgs Funnel has a dominant higgsino LSP, though only $0.5 \%$ are pure higgsino. The upper right plot space of Fig. 3 clearly shows the HF region with $M\left(H^{0} / A^{0}\right)$ plot as a function of the LSP $\widetilde{\chi}_{1}^{0}$. The minimum heavy Higgs boson mass in the HF region is $M\left(H^{0} / A^{0}\right) \sim 800 \mathrm{GeV}$, and these $H^{0} / A^{0}$ near this lower boundary all have $30 \leq \tan \beta \leq 40$, and given a branching ratio of more than $85 \%$ for $H^{0} / A^{0} \rightarrow b \bar{b}$, the lightest $M\left(H^{0} / A^{0}\right)$ in our model space persist comfortably beyond current LHC constraints for BSM Higgs searches [60].

In our light stau coannihilation strip, only $27.6 \%$ of the points have stau NLSP, with the remaining $72.4 \%$ having a chargino $\tilde{\chi}_{1}^{ \pm}$NLSP. As one would expect though, four nearly degenerate sparticles $\left(\tilde{\chi}_{1}^{0}, \tilde{\chi}_{1}^{ \pm}, \tilde{\chi}_{2}^{0}, \widetilde{\tau}_{1}^{ \pm}\right)$greatly suppresses the relic density, hence only the stau $\widetilde{\tau}_{1}^{ \pm}$NLSP points with larger $\tilde{\chi}_{1}^{ \pm}$and $\tilde{\chi}_{2}^{0}$ in the light stau coannihilation strip can generate the observed WMAP9 and Planck relic density. The light stau coannihilation strip is evident in the lower left panel of Fig. 2. We inspect the most recent ATLAS slepton con- straints [59] on compressed spectra, such as we have in the STAUC region, therefore we attempt to approximately emulate the ATLAS slepton exclusions by applying the following constraints on our model space, where both conditions for each must be TRUE for the point to be excluded: (1) $M\left(\tilde{\tau}_{1}^{ \pm}\right)<100 \mathrm{GeV}$ and $M\left(\tilde{\tau}_{1}^{ \pm}\right)-M\left(\tilde{\chi}_{1}^{0}\right)>1 \mathrm{GeV} ;(2)$ $100 \leq M\left(\tilde{\tau}_{1}^{ \pm}\right)<150 \mathrm{GeV}$ and $2 \leq M\left(\widetilde{\tau}_{1}^{ \pm}\right)-M\left(\tilde{\chi}_{1}^{0}\right) \leq$ $14 \mathrm{GeV}$; and (3) $150 \leq M\left(\tilde{\tau}_{1}^{ \pm}\right)<190 \mathrm{GeV}$ and $3 \leq$ $M\left(\tilde{\tau}_{1}^{ \pm}\right)-M\left(\tilde{\chi}_{1}^{0}\right) \leq 8 \mathrm{GeV}$. These restrictions are included in Figs. 1, 2, 3 and 4. Notice in constraint (1) just above that we allow for an off-shell tau lepton $\tau^{ \pm}$less than $1 \mathrm{GeV}$, an action that does capture a not insignificant number of viable points, in view of the fact that $10.5 \%$ of the STAUC region has $M\left(\tilde{\tau}_{1}^{ \pm}\right)-M\left(\tilde{\chi}_{1}^{0}\right)<1 \mathrm{GeV}$. A quick review of Table 4 indicates that the stau coannihilation spectra with light stau NLSP and $M(\widetilde{g})-M\left(\widetilde{t}_{1}\right)>M_{t}$ produce exclusively a 4-top signature $\left(\widetilde{g} \rightarrow \widetilde{t}_{1} t \rightarrow t \bar{t}+\widetilde{\chi}_{1}^{0}\right)$, giving rise to large multijet events, as is typical in $\mathcal{F}-S U(5)$ [20]. The final states are not as clear when $M(\widetilde{g})-M\left(\widetilde{t}_{1}\right)<M_{t}$ or $M(\widetilde{g})<M\left(\widetilde{t}_{1}\right)$ given that there is no dominant decay channel in either of these two cases. The model space is generally split between these two situations, where about half the STAUC region has $M(\widetilde{g})-M\left(\tilde{t}_{1}\right)>M_{t}$ and the other half of the STAUC region has $M(\widetilde{g})-M\left(\widetilde{t}_{1}\right)<M_{t}$ or $M(\widetilde{g})<M\left(\widetilde{t}_{1}\right)$. 


\section{Conclusion}

With our motivation partially inspired by D-brane model building, we presented an analysis of non-zero general SUSY breaking soft terms in $\mathcal{F}-S U(5)$. The methodology involved massive parallel computing given the large number of unknown parameters. While the resulting viable parameter space was extraordinarily constrained given the large number of computations, several interesting regions were uncovered that can simultaneously generate the WMAP and Planck observed relic density measurements and correct light Higgs boson mass, over and above satisfying many LHC search constraints. Four regions that accomplish these rely upon (1) light stop coannihilation, (2) pure Higgsino dark matter, (3) Higgs funnel, and (4) light stau coannihilation, though the pure Higgsino LSP could not reach the observed relic density due to its large annihilation cross-section. Concluding the analysis was an effort to identify the decay modes to final states that could represent observable signatures of these four scenarios, discovering that the light stop coannihilation and light stau coannihilation decay channels possess very dominant branching fractions. The light stop coannihilation produces the typical top+charm quark final state via the gluino decay and the light stau coannihilation mostly leads to the characteristic $\mathcal{F}-S U(5)$ large multijet event, whereas the pure Higgsino LSP and Higgs funnel provide no dominant decay channel to final states. Though possibly furnishing the highest intrigue, our exploration tentatively revealed that the $\mathcal{F}$-SU (5) model may indeed harbor a rather diminutive region exhibiting spectra with a mixed scenario of a dominant Higgsino LSP and light stop coannihilation, a rare yet rather natural SUSY spectrum, but that is the focus of our next endeavor.

Acknowledgements Portions of this research were conducted with high performance computational resources provided by the Louisiana Optical Network Infrastructure (http://www.loni.org). This research was supported in part by the Projects 11475238, 11647601, and 11875062 supported by the National Natural Science Foundation of China (TL), and by the DOE grant DE-FG02-13ER42020 (DVN).

Open Access This article is distributed under the terms of the Creative Commons Attribution 4.0 International License (http://creativecomm ons.org/licenses/by/4.0/), which permits unrestricted use, distribution, and reproduction in any medium, provided you give appropriate credit to the original author(s) and the source, provide a link to the Creative Commons license, and indicate if changes were made. Funded by SCOAP ${ }^{3}$.

\section{References}

1. G. Aad et al., (ATLAS), Observation of a new particle in the search for the Standard Model Higgs boson with the ATLAS detector at the LHC. Phys. Lett. B 716, 1 (2012). arXiv:1207.7214
2. S. Chatrchyan et al., (CMS), Observation of a new boson at a mass of $125 \mathrm{GeV}$ with the CMS experiment at the LHC. Phys. Lett. B 716, 30 (2012). arXiv: 1207.7235

3. M. Carena, S. Gori, N.R. Shah, C.E.M. Wagner, A $125 \mathrm{GeV}$ SMlike Higgs in the MSSM and the $\gamma \gamma$ rate. JHEP 03, 014 (2012). arXiv: 1112.3336

4. W. Adam, Searches for SUSY, Talk at the 38th International Conference on High Energy Physics, (2016)

5. S.M. Barr, A new symmetry breaking pattern for $S O(10)$ and proton decay. Phys. Lett. B 112, 219 (1982)

6. J.P. Derendinger, J.E. Kim, D.V. Nanopoulos, Anti-SU(5). Phys. Lett. B 139, 170 (1984)

7. I. Antoniadis, J.R. Ellis, J.S. Hagelin, D.V. Nanopoulos, Supersymmetric flipped $S U(5)$ revitalized. Phys. Lett. B 194, 231 (1987)

8. J. Jiang, T. Li, D.V. Nanopoulos, Testable flipped $S U(5) \times U(1)_{X}$ models. Nucl. Phys. B 772, 49 (2007). arXiv:hep-ph/0610054

9. J. Jiang, T. Li, D.V. Nanopoulos, D. Xie, F-SU(5). Phys. Lett. B 677, 322 (2009). arXiv:0811.2807

10. J. Jiang, T. Li, D.V. Nanopoulos, D. Xie, Flipped $S U(5) \times$ $U(1)_{X}$ models from F-theory. Nucl. Phys. B 830, 195 (2010). arXiv:0905.3394

11. J.L. Lopez, D.V. Nanopoulos, K.-J. Yuan, The search for a realistic flipped SU(5) string model. Nucl. Phys. B 399, 654 (1993). arXiv:hep-th/9203025

12. T. Li, J.A. Maxin, D.V. Nanopoulos, J.W. Walker, A Higgs mass shift to $125 \mathrm{GeV}$ and a multi-jet supersymmetry signal: miracle of the flippons at the $\sqrt{s}=7 \mathrm{TeV}$ LHC. Phys. Lett. B 710, 207 (2012a). arXiv: 1112.3024

13. Y. Huo, T. Li, D.V. Nanopoulos, C. Tong, The lightest CP-even Higgs Boson mass in the testable flipped $S U(5) \times U(1)_{X}$ models from F-theory. Phys. Rev. D 85, 116002 (2012). arXiv:1109.2329

14. T. Li, D.V. Nanopoulos, J.W. Walker, Fast proton decay. Phys. Lett. B 693, 580 (2010). arXiv:0910.0860

15. T. Li, D.V. Nanopoulos, J.W. Walker, Elements of fast proton decay. Nucl. Phys. B 846, 43 (2011a). arXiv:1003.2570

16. E. Cremmer, S. Ferrara, C. Kounnas, D.V. Nanopoulos, Naturally vanishing cosmological constant in $N=1$ supergravity. Phys. Lett. B 133, 61 (1983)

17. T. Li, J.A. Maxin, D.V. Nanopoulos, J.W. Walker, The golden point of no-scale and no-parameter $\mathcal{F}-S U(5)$. Phys. Rev. D 83, 056015 (2011b). arXiv: 1007.5100

18. T. Li, J.A. Maxin, D.V. Nanopoulos, J.W. Walker, The golden strip of correlated Top Quark, Gaugino, and vectorlike mass in no-scale, no-parameter $\mathcal{F}-S U(5)$. Phys. Lett. B 699, 164 (2011c). arXiv:1009.2981

19. T. Li, J.A. Maxin, D.V. Nanopoulos, J.W. Walker, The unification of dynamical determination and bare minimal phenomenological constraints in no-scale $\mathcal{F}$-SU (5). Phys. Rev. D 85, 056007 (2012b). arXiv: 1105.3988

20. T. Li, J.A. Maxin, D.V. Nanopoulos, J.W. Walker, The ultrahigh jet multiplicity signal of stringy no-scale $\mathcal{F}-S U(5)$ at the $\sqrt{s}=7$ TeV LHC. Phys. Rev. D 84, 076003 (2011d). arXiv:1103.4160

21. G. Giudice, A. Masiero, A natural solution to the mu problem in supergravity theories. Phys. Lett. B 206, 480 (1988)

22. T. Leggett, T. Li, J. A. Maxin, D. V. Nanopoulos, J. W. Walker, No naturalness or fine-tuning problems from no-scale supergravity (2014), arXiv:1403.3099

23. T. Leggett, T. Li, J.A. Maxin, D.V. Nanopoulos, J.W. Walker, Confronting electroweak fine-tuning with no-scale supergravity. Phys. Lett. B 740, 66 (2015). arXiv: 1408.4459

24. T. Li, J.A. Maxin, D.V. Nanopoulos, The return of the king: noscale $\mathcal{F}-S U(5)$. Phys. Lett. B764, 167 (2017a). arXiv:1609.06294

25. T. Li, J.A. Maxin, D.V. Nanopoulos, Probing the No-Scale $\mathcal{F}$ $S U$ (5) one-parameter model via gluino searches at the LHC2. Phys. Lett. B773, 54 (2017b). arXiv:1705.07973 
26. C.-M. Chen, T. Li, D.V. Nanopoulos, Flipped and unflipped SU(5) as type IIA flux vacua. Nucl. Phys. B 751, 260 (2006). arXiv:hep-th/0604107

27. T. Li, J.A. Maxin, D.V. Nanopoulos, J.W. Walker, No-scale $\mathcal{F}$ $S U(5)$ in the light of LHC, planck and XENON. J. Phys. G 40, 115002 (2013). arXiv:1305.1846

28. ATLAS, Exotics Combined Summary Plots. https://twiki.cern. ch/twiki/bin/view/AtlasPublic/ExoticsPublicResults. Accessed 20 Nov 2018

29. R. Harnik, D.T. Larson, H. Murayama, M. Thormeier, Probing the Planck scale with proton decay. Nucl. Phys. B 706, 372 (2005). arXiv:hep-ph/0404260

30. J.R. Ellis, D.V. Nanopoulos, K.A. Olive, Flipped heavy neutrinos: from the solar neutrino problem to baryogenesis. Phys. Lett. B 300, 121 (1993a). arXiv:hep-ph/9211325

31. J.R. Ellis, J.L. Lopez, D.V. Nanopoulos, K.A. Olive, Flipped angles and phases: a systematic study. Phys. Lett. B 308, 70 (1993b). arXiv:hep-ph/9303307

32. T. Li, J.A. Maxin, D.V. Nanopoulos, J.W. Walker, Dark matter, proton decay and other phenomenological constraints in $\mathcal{F}$-SU(5). Nucl. Phys. B 848, 314 (2011e). arXiv: 1003.4186

33. T. A., Aaltonen (collaboration Tevatron Electroweak Working Group, CDF, D0), Combination of CDF and DO results on the mass of the top quark using up to $8.7 \mathrm{fb}^{-1}, 2013$. arXiv: 1305.3929

34. G. Hinshaw et al., (collaboration WMAP), Nine-year Wilkinson microwave anisotropy probe (WMAP) Observations: cosmological parameter results. Astrophys. J. Suppl. 208, 19 (2013). arXiv: 1212.5226

35. P. A. R. Ade et al. (collaboration Planck), Planck 2015 results. XIII. Cosmological parameters. Astron. Astrophys. 594, A13 (2016). arXiv: 1502.01589

36. N. Aghanim et al. (collaboration Planck), Planck 2018 results. VI. Cosmological parameters (2018). arXiv: 1807.06209

37. HFAG, (2013). http://www.slac.stanford.edu/xorg/hfag/rare/ 2013/radll/OUTPUT/TABLES/radll.pdf

38. V. Khachatryan et al., (collaboration LHCb, CMS), Observation of the rare $B_{s}^{0} \rightarrow \mu^{+} \mu^{-}$decay from the combined analysis of CMS and LHCb data. Nature 522, 68 (2015). arXiv: 1411.4413

39. T. Aoyama, M. Hayakawa, T. Kinoshita, M. Nio, Complete tenthorder QED contribution to the Muon g-2. Phys. Rev. Lett. 109, 111808 (2012). arXiv: 1205.5370

40. D.S. Akerib et al., Results from a search for dark matter in LUX with 332 live days of exposure (2016). arXiv: 1608.07648

41. A. Tan et al. (collaboration PandaX-II), Dark matter results from first 98.7-day data of PandaX-II experiment. Phys. Rev. Lett. 117, 121303 (2016). arXiv: 1607.07400

42. E. Aprile et al., (XENON), Dark matter, search results from a one tonne $\times$ year exposure of XENON1T. arXiv:1805.12562 (2018)

43. E. Behnke et al., (COUPP), First dark matter search results from a 4-kg CF 3 I bubble chamber operated in a deep underground site. Phys. Rev. D86, 052001 (2012). arXiv:1204.3094 (note [Erratum: Phys. Rev. D90, no. 7, 079902 (2014)])

44. E. Aprile et al., (XENON100), Limits on spin-dependent WIMPnucleon cross sections from 225 live days of XENON100 data. Phys. Rev. Lett. 111, 021301 (2013). arXiv:1301.6620
45. V. Takhistov (Super-Kamiokande), in booktitle Proceedings, 51st Rencontres de Moriond on Electroweak Interactions and Unified Theories: La Thuile, Italy, March 12-19, 2016 (2016), pp. 437444. arXiv: 1605.03235

46. G. Belanger, F. Boudjema, A. Pukhov, A. Semenov, Dark matter direct detection rate in a generic model with micrOMEGAs2.1, Comput. Phys. Commun. 180, 747 (2009). arXiv:0803.2360

47. A. Djouadi, J.-L. Kneur, G. Moultaka, SuSpect: a Fortran code for the supersymmetric and Higgs particle spectrum in the MSSM. Comput. Phys. Commun. 176, 426 (2007a). arXiv:hep-ph/0211331

48. A. Djouadi, M.M. Muhlleitner, M. Spira, Decays of supersymmetric particles: The Program SUSY-HIT (SUspect-SdecaYHdecay-InTerface). Acta Phys. Pol. B 38, 635 (2007b). arXiv:hep-ph/0609292

49. C. Patrignani et al., (Particle Data Group), Review of particle physics. Chin. Phys. C 40, 100001 (2016)

50. R. De Benedetti, T. Li, J. A. Maxin, D. V. Nanopoulos (2018) (note in preparation)

51. M. Aaboud et al., (ATLAS), Search for dark matter and other new phenomena in events with an energetic jet and large missing transverse momentum using the ATLAS detector. JHEP 01, 126 (2018a). arXiv: 1711.03301

52. M. Aaboud et al., (ATLAS), Search for supersymmetry in final states with charm jets and missing transverse momentum in $13 \mathrm{TeV}$ $p p$ collisions with the ATLAS detector (2018b). arXiv:1805.01649

53. A. M. Sirunyan et al., (CMS), Search for the pair production of third-generation squarks with two-body decays to a bottom or charm quark and a neutralino in proton-proton collisions at $\sqrt{s}$ = 13 TeV. Phys. Lett. 263(2018a). arXiv:1707.07274 (B778,)

54. A. M. Sirunyan et al., (CMS), Search for new phenomena with the $M_{\mathrm{T} 2}$ variable in the all-hadronic final state produced in protonproton collisions at $\sqrt{s}=13 \mathrm{TeV}$, Eur. Phys. J. C77, 710 (2017a). arXiv: 1705.04650

55. A. M. Sirunyan et al., (CMS), Search for direct production of supersymmetric partners of the top quark in the all-jets final state in proton-proton collisions at $\sqrt{s}=13 \mathrm{TeV}$. JHEP 10, 005 (2017b). arXiv: 1707.03316

56. A. M. Sirunyan et al., (CMS), Search for natural and split supersymmetry in proton-proton collisions at $\sqrt{s}=13 \mathrm{TeV}$ in final states with jets and missing transverse momentum. JHEP 05, 025 (2018b). arXiv: 1802.02110

57. J. Ellis, J. L. Evans, F. Luo, K. A. Olive, J. Zheng, Stop coannihilation in the CMSSM and SubGUT models. Eur. Phys. J. C78, 425 (2018). arXiv: 1801.09855

58. W. Ahmed, L. Calibbi, T. Li, S. Raza, J.-S. Niu, and X.-C. Wang, Naturalness and dark matter in a realistic intersecting D6-brane model. JHEP 06, 126 (2018). arXiv:1711.10225

59. M. Aaboud et al., (ATLAS), Search for electroweak production of supersymmetric states in scenarios with compressed mass spectra at $\sqrt{s}=13 \mathrm{TeV}$ with the ATLAS detector. Phys. Rev. D 97, 052010 (2018c). arXiv:1712.08119

60. A. M. Sirunyan et al., (CMS), Search for beyond the standard model Higgs bosons decaying into a $b \bar{b}$ pair in pp collisions at $\sqrt{s}=13$ $\mathrm{TeV}$ (2018c). arXiv:1805.12191 